\title{
MACROMICETOS LIGNÍCOLAS DE LA SIERRA NORTE DE PUEBLA, MÉXICO, CON NOTAS SOBRE SU DISTRIBUCIÓN ALTITUDINAL
}

\author{
Sadoth Vázquez ${ }^{1,3}$, Ricardo Valenzuela ${ }^{2}$ y Rafael F. del Castillo ${ }^{1}$ \\ ${ }^{1}$ Intituto Politécnico Nacional, Centro Interdisciplinario de Investigación para el \\ Desarrollo Integral Regional, Unidad Oaxaca, Avenida Hornos 1003, \\ 71230 Sta. Cruz Xoxocotlán, Oaxaca, México. \\ ${ }^{2}$ Instituto Politécnico Nacional, Escuela Nacional de Ciencias Biológicas, \\ Laboratorio de Micología, Plan de Ayala s/n., Colonia Casco de Santo Tomás, \\ 02020 México, D.F., México. \\ 3Autor para la correspondencia: savazq70@hotmail.com
}

\section{RESUMEN}

Se presenta un listado preliminar de hongos lignícolas de la Sierra Norte de Puebla, Puebla, México. Para ello se realizaron ocho exploraciones en los años 2009 y 2010, además de efectuar la revisión de todos los especímenes provenientes del área de estudio depositados en la colección de hongos del herbario de la Escuela Nacional de Ciencias Biológicas, Instituto Politécnico Nacional. Se analiza también la distribución altitudinal y por tipo de vegetación de los especímenes encontrados. Un total de 176 especímenes distribuidos en 95 especies fueron encontrados para la zona; 39 son nuevos registros para la región y un espécimen probablemente corresponda a una nueva especie. Los resultados indican la importancia de continuar realizando inventarios fúngicos para establecer bases confiables para posteriores estudios ecológicos y poder proponer planes de conservación para los hongos lignícolas.

Palabras clave: Ascomycota, Basidiomycota, biodiversidad.

\section{ABSTRACT}

We present a preliminary checklist of lignicolous macrofungi from the Sierra Norte de Puebla, Puebla, Mexico. For this purpose, we conducted eight field trips during 2009 and 2010, and reviewed all specimens from the study area deposited in the fungus 
collection of the herbarium of the Escuela Nacional de Ciencias Biológicas, Instituto Politécnico Nacional. In addition, we analyzed altitudinal distribution as well as for vegetation type. A total of 176 specimens belonging to 95 species were recorded for the zone; 39 are new records for the region, and one specimen probably is a new species. The results indicate the importance of continuing to carry out fungal inventories that could be a reliable base for future ecological studies and to propose conservation plans for wood decay fungi.

Key words: Ascomycota, Basidiomycota, biodiversity.

\section{INTRODUCCIÓN}

El estado de Puebla se localiza en la región este de México, está dividido políticamente en 217 municipios de los cuales 40 conforman la región Sierra Norte. Debido a su abrupto gradiente altitudinal y a la consecuente diversidad de condiciones ambientales, la Sierra Norte de Puebla presenta gran diversidad de tipos de vegetación, desde selvas medianas hasta bosques templados y fríos (Martínez et al., 2007).

En el aspecto micológico, la Sierra Norte de Puebla es casi terra ignota. Si bien existen numerosos estudios que reportan especies de hongos para esta zona del estado (García Romero et al., 1970; Guzmán y Herrera, 1971; Pérez-Silva, 1973, 1975, 1981; Guzmán et al., 1975; Chacón y Guzmán, 1983; Bandala-Muñoz et al., 1988; Medel et al., 1999; Medel, 2002; Valenzuela et al., 2004, 2005; Medel et al., 2010), los trabajos específicos para la Sierra Norte son escasos. Martínez-Alfaro et al. (1983) realizaron un análisis etnomicológico general y Vázquez-Mendoza y Valenzuela-Garza (2010) elaboraron un listado de los macromicetos presentes en la región de interés. No se encontró ningún trabajo específico respecto a hongos lignícolas para el área de estudio.

Debido a que la Sierra Norte de Puebla se encuentra entre dos áreas de conocida riqueza fúngica, las regiones norte de los estados de Hidalgo y Veracruz (Villaruel-Ordaz y Cifuentes, 2007; Medel et al., 2008; Romero Bautista et al., 2010), se espera que la diversidad de macromicetos en ella sea también alta.

El objetivo del presente estudio es establecer un inventario que sirva como línea basal para el conocimiento de los hongos degradadores de madera existentes en la región Sierra Norte del estado de Puebla, por medio de listados de especies de estos organismos, así como presentar algunas notas respecto a su distribución. 


\section{MATERIAL Y MÉTODO}

Se efectuó la revisión del total de especímenes provenientes del área de estudio depositados en la colección de hongos del herbario de la Escuela Nacional de Ciencias Biológicas, Instituto Politécnico Nacional. Se corroboraron datos de identificación y/o localidad de los ejemplares. Adicionalmente se realizaron ocho salidas de colecta a cuatro localidades en los municipios de Zapotitlán de Méndez, Zacapoaxtla, y Chignahuapan, en los años 2009 y 2010. La caracterización morfológica de los ejemplares colectados se hizo de acuerdo con las técnicas indicadas por Cifuentes et al. (1986). Para la identificación de los especímenes se utilizaron los trabajos de Erikssonet al. (1978), Ryvarden y Johansen (1980), Marmolejo et al. (1981), Gilbertson y Ryvarden (1986, 1987), León-Gómez y Pérez-Silva (1988) y Parmasto (2001). Todos los ejemplares fueron depositados en el herbario antes mencionado.

Por otra parte se registraron datos de vegetación y altitud para los especímenes colectados, verificándolos mediante el uso de cartas topográficas para los ejemplares de herbario (INEGI, 1983; 1984; 1998, 1999 a, b, c). El intervalo altitudinal detectado fue de 500 a $3000 \mathrm{~m}$, mismo que en los análisis de distribución fue dividido en franjas de $500 \mathrm{~m}$.

\section{RESULTADOS}

Se revisaron un total de 176 especímenes de hongos lignícolas correspondientes a 95 especies: 11 pertenecen al Phylum Ascomycota y 84 a Basidiomycota. Se reportan 39 nuevos registros para la región norte del estado de Puebla y una probable nueva especie del género Hymenochaete, la cual se encuentra en proceso de descripción. Los órdenes mejor representados fueron Polyporales, Hymenochaetales y Stereales con 37, 15 y 8 especies respectivamente. Las familias con mayor número de especies fueron Polyporaceae con 33, Hymenochaetaceae con 15 y Stereaceae con 7. Los géneros mejor representados fueron Hymenochaete, Stereum, Trametes y Trichaptum, con seis especies cada uno. El listado de macromicetos lignícolas se presenta con arreglo alfabético (Apéndice). Los siguientes datos se dan para cada espécimen: colector, fecha de colecta y localidad (Fig.1).

Los géneros y especies se reportan siguiendo la clasificación modificada de Kirk et al. (2008). Las novedades en la distribución de los organismos se indican por: ${ }^{*}=$ nuevo registro para la región; $+=$ nueva especie. 

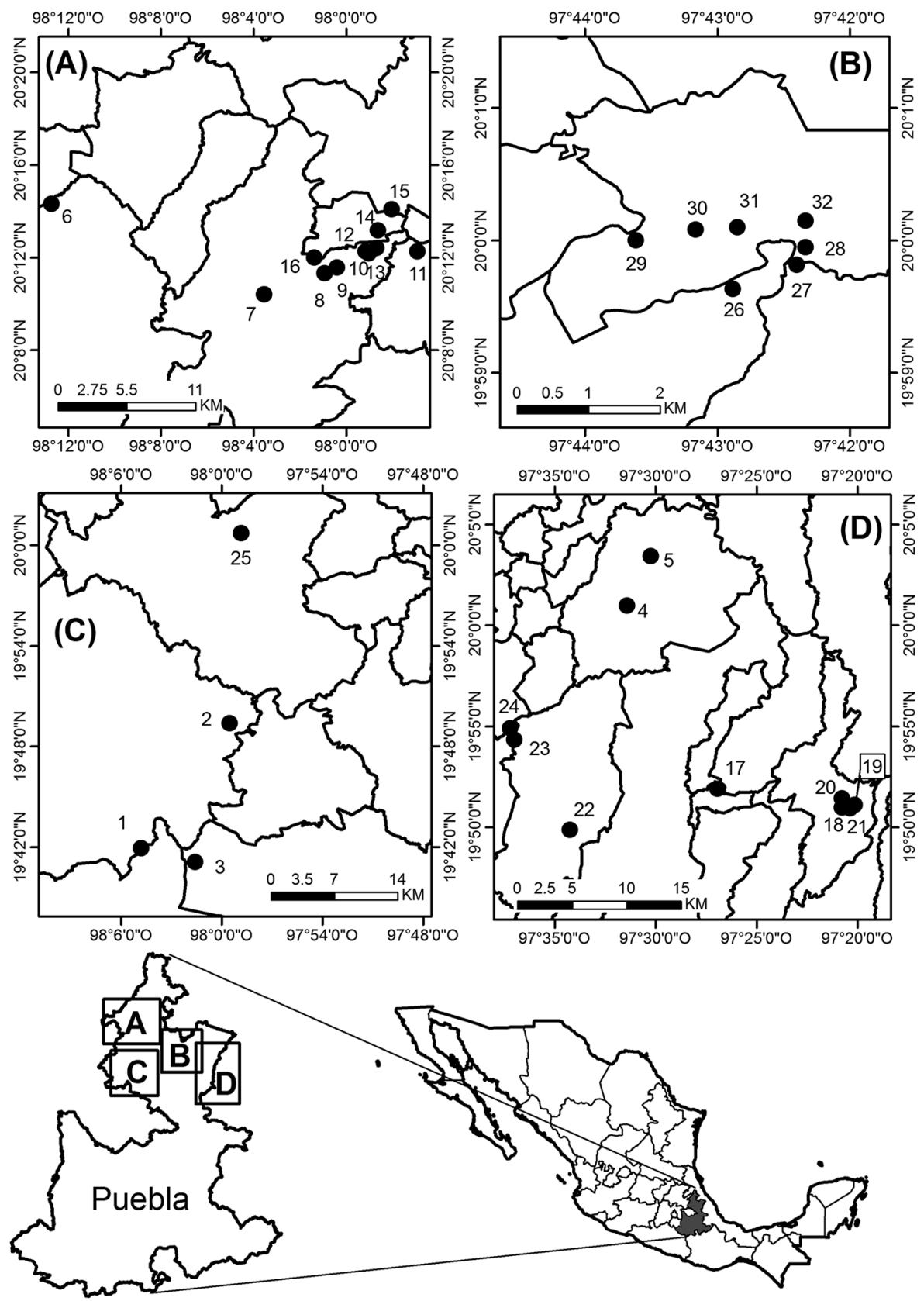

Fig. 1. Ubicación de localidades de colecta por municipio. A. Honey: 6; Huauchinango, 7 a 9; Juan Galindo, 10 a 16. B. Zapotitlán de Méndez, 26 a 32. C. Chignahuapan, 1 a 3; Zacatlán, 25. D. Cuetzalan, 4 y 5; Tételes, 17; Teziutlán: 18 a 21; Zacapoaxtla, 22 a 24. 
La distribución altitudinal de las especies muestra dos puntos máximos de riqueza específica y parece indicar un patrón cuadrático de la misma (Fig. 2). Por otra parte, el análisis de distribución por tipo de vegetación muestra un mayor número de especies en los bosques templados (Fig. 3).

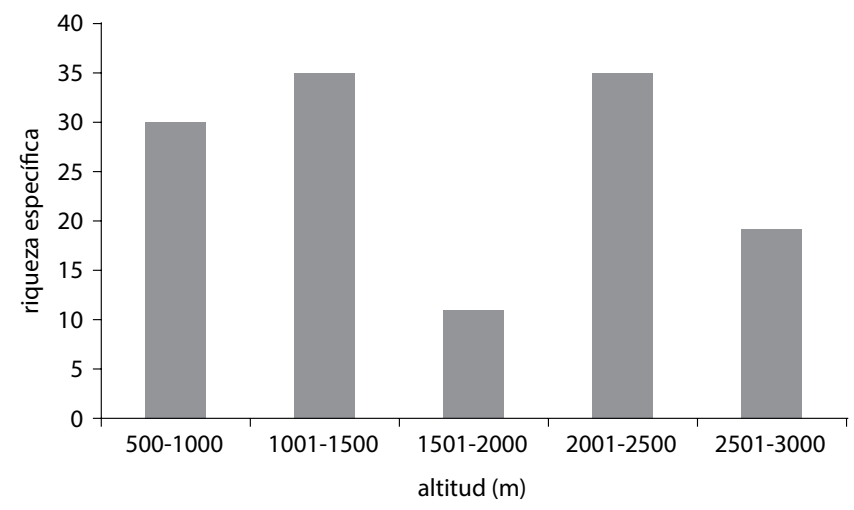

Fig. 2. Distribución altitudinal para las especies de hongos lignícolas en la Sierra Norte de Puebla.

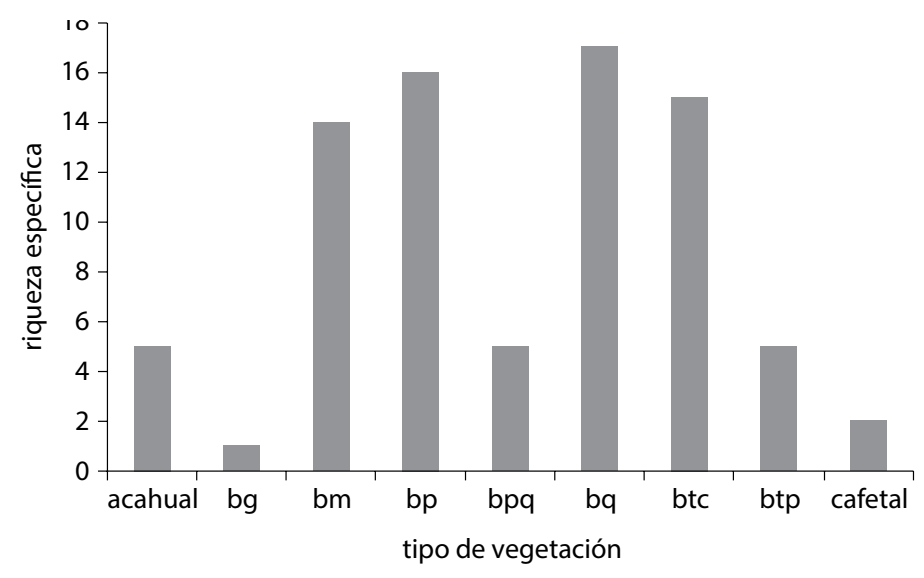

Fig. 3. Distribución por tipo de vegetación de los hongos lignícolas en Sierra Norte de Puebla. bg: bosque de galería, bm: bosque mesófilo, bp: bosque de pino, bpq: bosque de pino encino, bq: bosque de encino, btc: bosque tropical caducifolio, btp: bosque tropical perennifolio. 


\section{DISCUSIÓN}

La Sierra Norte de Puebla presenta una alta diversidad fúngica, similar a la reportada para las áreas vecinas de los estados de Hidalgo y Veracruz (Romero Bautista et al., 2010; Vázquez-Mendoza y Valenzuela-Garza, 2010). Los órdenes mejor representados fueron similares a los registrados para otras regiones del país, tanto en familias como en el número de especies (Salinas-Salgado et al., 2012). El reporte de una nueva especie y los 39 nuevos registros para la región señalan la importancia de incrementar las exploraciones micológicas para complementar los inventarios fúngicos, en especial si se considera que estos reportes provienen de los especímenes colectados en campo.

El análisis altitudinal parece mostrar un patrón cuadrático, aunque es necesario incrementar el esfuerzo de colecta en especial en la franja entre 1500 y 2000 $\mathrm{m}$, para establecer el patrón real. La tendencia mostrada es contraria a lo reportado para otros grupos de organismos, donde se observan patrones monotónicos negativos (Navarro, 1992; Jacquemyn et al., 2005). Lo anterior señala diferencias entre la distribución altitudinal de los hongos lignícolas con respecto a la de otros taxa. Al no considerarse estas diferencias en los distintos planes de conservación, se estarían dejando fuera de ellos a grupos tan importantes y altamente diversos como son los hongos lignícolas.

La distribución por tipo de vegetación muestra una mayor riqueza específica en bosques templados, concordante con lo reportado por Vázquez-Mendoza y Valenzuela-Garza (2010) para macromicetos en general para la misma región de Puebla, así como por García-Jiménez y Garza-Ocañas (2001) para boletáceos en el norte del país. Sin embargo, será necesario implementar protocolos de colecta a futuro que consideren estos factores (diversidad de hospederos, factores climáticos) para así tener una mejor visión de los patrones de distribución de los organismos, que nos ayuden a establecer medidas adecuadas de conservación de los mismos.

En conclusión, es necesario aumentar el número de trabajos sobre investigación básica (inventarios, distribución, etc.) para los hongos lignícolas en el estado de Puebla. El desconocimiento de estos aspectos para los recursos presentes en una región, en este caso hongos degradadores de madera, dificulta la propuesta y aplicación de planes de aprovechamiento y conservación. Se hace imperante desarrollar estudios ecológicos que permitan tratar de entender los patrones de distribución de las especies, en especial si se busca establecer programas de conservación a largo plazo. 


\section{AGRADECIMIENTOS}

Los autores agradecen al Instituto Politécnico Nacional por los apoyos para la realización de este trabajo a través del proyecto SIP 20110385. Vázquez también agradece a las autoridades civiles de los municipios visitados por su apoyo para realizar las colectas; y al Biól. Raúl Rivera García, del Laboratorio de Sistemas de Información Geográfica del Centro Interdisciplinario de Investigación para el Desarrollo Integral Regional Unidad Oaxaca, por su ayuda para elaborar el mapa de localidades.

\section{LITERATURA CITADA}

Bandala Muñoz, V. M., G. Guzmán y L. Montoya Bello. 1988. Especies de macromicetos citadas de México VII. Agaricales, parte II (1972-1987). Rev. Mex. Mic. 4: 205-250.

Chacón, S. y G. Guzmán. 1983. Especies de macromicetos citadas de México V. Ascomycetes, parte II. Bol. Soc. Mex. Mic. 18: 103-114.

Cifuentes, J., M. Villegas y L. Pérez-Ramírez. 1986. Hongos. In: Lot, A. y F. Chiang (Eds.). Manual de herbario. Consejo Nacional de la Flora de México, A.C. México, D.F. México. pp. 55-64.

Eriksson, J., K. Hjörtstam y L. Ryvarden.1978. The Corticiaceae of North Europe, Vol. 5 Mycoaciella-Phanerochaete. Fungiflora. Oslo, Noruega. pp. 887-1048.

García Jiménez, J. y F. Garza Ocañas.2001. Conocimiento de los hongos de la familia Boletaceae de México. Ciencia UANL 14(3): 336-344.

García Romero, L., G. Guzmán y T. Herrera.1970. Especies de macromicetos citados de México I. Ascomycetos, Tremellales y Aphyllophorales. Bol. Soc. Mex. Mic. 4: 5476.

Gilbertson, R. L. y L. Ryvarden.1986. North American polypores. Abortiporus- Lindtneria. Vol. 1. Fungiflora. Oslo, Noruega. 443 pp.

Gilbertson, R. L. y L. Ryvarden.1987. North American polypores. MegasporoniaWrightporia. Vol. 2. Fungiflora. Oslo, Noruega. 843 pp.

Guzmán, G. y T. Herrera. 1971. Especies de macromicetos citadas de México II. Fistulinaceae, Meruliaceae y Polyporaceae. Bol.Soc. Mex. Mic. 5: 57-78.

Guzmán, G., R. G. Wasson y T. Herrera. 1975. Una iglesia dedicada al culto de un hongo, "Nuestro Señor del Honguito" en Chignahuapan, Puebla. Bol. Soc. Mex. Mic. 9: 137-147.

INEGI. 1983. Carta Topográfica 1:50000, Chignahuapan, clave E14B13. INEGI, México.

INEGI. 1984. Carta Topográfica 1:50000, Pahuatlán, clave F14D73. INEGI, México.

INEGI. 1998. Carta Topográfica 1:50000, Zacatlán, clave E14B14. INEGI, México.

INEGI. 1999a. Carta Topográfica 1:50000, Cuetzalan, clave F14D85. INEGI, México.

INEGI. 1999b. Carta Topográfica 1:50000, Huauchinango, clave F14D83. INEGI, México.

INEGI. 1999c. Carta Topográfica 1:50000, Teziutlán, clave E14B15. INEGI, México. 
Jacquemyn, H., C. Micheneau, D. L. Roberts y T. Pailler. 2005. Elevational gradients of species diversity, breeding system and floral traits of orchid species on Reunion Island. J. Biogeogr. 32: 1751-1761.

Kirk, P. M., P. F. Cannon, D. W. Minter y J. A. Stalpers. 2008. Ainsworth and Bisby's Dictionary of the Fungi. 10 ed. International Mycological Institute, CAB International. Wallingford, UK. $784 \mathrm{pp}$.

León-Gómez, C. y E. Pérez-Silva.1988. Especies de Nidulariales (Gasteromycetes) comunes en México. Rev. Mex. Mic. 4: 161-184.

Marmolejo, J. G., J. Castillo y G. Guzmán.1981. Descripción de las especies de Teleforáceos poco conocidos en México. Bol. Soc. Mex. Mic. 15: 9-63.

Martínez-Alfaro, M. A., E. Pérez-Silva y E. Aguirre-Acosta.1983. Etnomicología y exploraciones micológicas en la Sierra Norte de Puebla. Bol. Soc. Mex. Mic. 18: 51-63.

Martínez, M. A., V. Evangelista, F. Basurto, M. Mendoza y A. Cruz-Rivas. 2007. Flora útil de los cafetales en la Sierra Norte de Puebla, México. Rev. Mex. Biodiv. 78: 15-40.

Medel, R. 2002. Nuevos registros de Pyrenomycetes (Ascomycotina) en México. Bol. Soc. Bot. Mex. 70: 79-85.

Medel, R., G. Guzmán y S. Chacón. 1999. Especies de macromicetos citadas de México IX. Ascomycetes Parte III: 1983-1996. Acta Bot. Mex. 46: 57-72.

Medel, R., R. Castillo y G. Guzmán. 2008. Las especies de Xylaria (Ascomycota, Xylariaceae) conocidas de Veracruz, México, y discusión de nuevos registros. Rev. Mex. Mic. 28: 101-118.

Medel, R., G. Guzmán y R. Castillo. 2010. Adiciones al conocimiento de Xylaria (Ascomycota, Xylariales) en México. Rev. Mex. Mic. 31: 9-18.

Navarro, A. G. 1992. Altitudinal distribution of birds in the Sierra Madre del Sur, Guerrero, Mexico. The Condor 94: 29-39.

Parmasto, E. 2001. Hymenochaetoid fungi (Basidiomycota) of North America. Mycotaxon 79: 107-176.

Pérez-Silva, E. 1973. El género Daldinia (Pyrenomycetes) en México. Bol. Soc. Mex. Mic. 7: 51-58.

Pérez-Silva, E. 1975. El género Xylaria (Pyrenomycetes) en México. I. Bol. Soc. Mex. Mic. 9: 31-52.

Pérez-Silva, E. 1981. Nuevos registros de Amanita de la sección Caesareae (Agaricales) para México. Bol. Soc. Mex. Mic. 16: 141-149.

Romero Bautista, L., G. Pulido-Flores y R. Valenzuela. 2010. Estudio micoflorístico de los hongos poliporoides del estado de Hidalgo, México. Polibotánica 29: 1-28.

Ryvarden, L. e I. Johansen. 1980. A preliminary polypore flora of east Africa. Fungiflora. Oslo, Noruega. 636 pp.

Salinas-Salgado, E., R. Valenzuela, T. Raymundo, M. Cipriano-Salazar, B. Cruz-Lagunas y E. Hernández-Castro. 2012. Macromicetos xilófagos del bosque tropical caducifolio en el municipio de Cocula, Guerrero, México. Polibotánica 34: 137-155.

Valenzuela, R., T. Raymundo y M. R. Palacios. 2004. Macromicetos que crecen sobre Abies religiosa en el eje neovolcánico transversal. Polibotánica 18: 33-52.

Valenzuela, R., T. Raymundo y J. Cifuentes. 2005. La Familia Hymenochaetaceae en México II. Especies poco conocidas del género Phellinus. Rev. Mex. Mic. 20: 13-19. 
Vázquez-Mendoza, S. y R. Valenzuela-Garza. 2010. Macromicetos de la Sierra Norte del Estado de Puebla, México. Naturaleza y Desarrollo 8(1): 46-61.

Villaruel-Ordaz, J. L. y J. Cifuentes. 2007. Macromicetos de la cuenca del Río Magdalena y zonas adyacentes. Delegación La Magdalena Contreras, México, D.F. Rev. Mex. Mic. 25: 59-68.

Recibido en enero de 2014. Reactivación en diciembre de 2014.

Aceptado en septiembre de 2015. 


\section{APÉNDICE}

Macromicetos lignícolas presentes en la Sierra Norte de Puebla, México, y especímenes examinados.

\section{ASCOMYCOTA}

Discomycetes

Leotiales

Leotiaceae

Leotia lubrica (Scop.) Pers.: M. Becerril Cg3, 07.11.2009, Loc. 1.

\section{Pezizales}

\section{Pyrenomataceae}

*Scutellinia scutellata (L.) Lambotte: $N$. Martinez Z-33: 28.11.2009, Loc. 22.

Tarzetta catinus (Holmsk.) Korf \& J.K. Rogers: S. Vázquez Cg-13: 08.04.2009, Loc. 1.

\section{Sarcoscyphaceae}

*Sarcoscypha aff. occidentalis (Schwein.) Sacc.: N. Martínez Z-3a: 28.11.2009, Loc. 22.

*Sarcoscypha coccinea (Jacq.) Sacc.: $N$. Martínez Z-3: 28.11.2009, Loc. 22; S. Vázquez Zx-38: 28.11.2009, Loc. 22.

\section{Pyrenomycetes}

Xylariales

Xylariaceae

*Daldinia concentrica (Bolton) Ces. \& De Not.: S. Vázquez Ap-3: 06.11.2009, Loc. 23.

*Hypoxylon aff. fragiforme: S. Vázquez Cg22: 08.04.2009, Loc. 1.

Hypoxylon thouarsianum (Leveille) Lloyd: G. Guzmán s.n.: 06.08.1967, Loc. 11.; S. Vázquez Zx-78: 13.03.2010, Loc. 22.

Poronia oedipus (Montagne) Montagne: $G$. Guzmán 2457: 23.07.1960, Loc. 12.

Xylaria cf. corniculata Sacc.: N. Martínez Z-26: 28.11.2009, Loc. 22.

Xylaria fockei (Mig.) Cooke: F. Ventura s.n.: 31.07.1970, Loc. 21.

\section{BASIDIOMYCOTA}

Hymenomycetes

Agaricales

Agaricaceae

Cyathus berkeleyanus (Tulasne) Lloyd: $B$. Olivares s.n.: 04.09.1966, Loc. 11.

Cyathus canna Lloyd: G. Guzmán 5401: 04.09.1966, Loc. 12.

Cyathus earlei Lloyd: R. Cruz s.n.: 29.07.1963, Loc. 14.

Inocybaceae

*Crepidotus mollis (Schaeff.) Staude: $S$. Vázquez Zx-36: 28.11.2009, Loc. 22.

\section{Mycenaceae}

*Xeromphalina tenuipes (Schwein.) A.H. Sm.: N. Martínez Z-25, 28.11.2009, Loc. 22.

\section{Schizophyllaceae}

Schizophyllum commune Fr.: $R$. Torres s.n.: 11.1979, Loc. 7.; J.G. García 21: 15.07.1979, Loc. 12.; R. Valenzuela 1290: 21.03.1983, Loc. 12; G. Rodríguez s.n.: 21.03.1983, Loc. 12. S. Vázquez Za34: 03.10.2009, Loc. 30; S. Vázquez Za36: 03.10.2009, Loc. 30; S. Vázquez Za83: 13.03.2010, Loc. 30; S. Vázquez Cg-68, 07.11.2009; Loc. 1.

\section{Strophariaceae}

Hypholoma fasciculare (Huds.) P. Kumm: N. Martínez Z-1: 28.11.2009, Loc. 22.

\section{Auriculariales}

Auriculariaceae

*Auricularia auricula-judae (Bull.) Quél.: S. Vázquez Za2: 03.10.2009, Loc. 30.; S. Vázquez Zx-10: 28.11.2009, Loc. 22; S. Vázquez Cg-3: 08.04.2009, Loc. 1.

Auricularia delicata Fr.: Vázquez \& Palacios 26: 23.10.1993, Loc. 29. 
Apéndice. Continuación.

Auricularia fuscosuccinea (Montagne)

Farlow: Vázquez \& Palacios 25: 23.10.1993, Loc. 29.

*Auricularia mesenterica (Dicks.) Pers.: $S$. Vázquez Za32: 03.10.2009, Loc. 30; S. Vázquez Zx-37: 28.11.2009, Loc. 22.

\section{Fistulinales}

\section{Fistulinaceae}

Fistulina radicata Schw.: P. Ramírez s.n.: 10.07.1981, Loc. 25.

\section{Hymenochaetales}

Hymenochaetaceae

Coltricia focicola (Berkeley et Curtis) Murrill: F. Ventura 1883: 30.07.1970, Loc. 21.

Coltricia montagnei (Fr.) Murrill: $G$. Guzmán 2177: sin fecha, Loc. 12.; A.

Lerma s.n.: 16.09.1979, Loc. 18.

Coltricia perennis (Fr.) Murrill: H. Ríos s.n.:

07.1967, Loc. 12; R. Cruz s.n.: 29.07.1963,

Loc. 15; Mille s.n., 06.08.1967, Loc. 11.

Cyclomyces iodinus (Montagne) Patouillard:

S. Vázquez \& Palacios 10: 23.10.1993, Loc. 29.

*Hydnochaete olivacea (Schwein.) Banker:

S. Vázquez Zx-82: 13.03.2010, Loc. 22.

*Hydnochaete tabacina (Berk \& M.A.

Curtis ex Fr.) Ryvarden: $S$. Vázquez Zx-

50: 13.03.2010, Loc. 22.

*Hymenochaete aff. jobii Parmasto: S.

Vázquez Cg-24: 08.04.2009, Loc. 1.

*Hymenochaete aff. rubiginosa (Dicks.) Lév.:

S. Vázquez Zx-74: 13.03.2010, Loc. 22.

Hymenochaete damicornis (Link) Lév.: $F$.

Ventura 1944: 31.07.1970, Loc. 21; F.

Ventura 2217: 28.08.1970, Loc. 21.

*Hymenochaete rhabarbarina (Berk.)

Cooke: S. Vázquez Ap-37: 31.03.2010,

Loc. 23; S. Vázquez Zx-51: 13.03.2010,

Loc. 22; S. Vázquez Zx-73: 13.03.2010,

Loc. 22.
Hymenochaete rheicolor (Mont.) Lév.: A.J. Sharp 3064: 24.03.1945, Loc. 7.

+*Hymenochaete sp. Z: S. Vázquez Zx-77: 13.03. 2010, Loc. 22.

*Inocutis jamaicensis Murrill: S. Vázquez Cg-36: 07.11.2009, Loc. 1.

Fuscoporia callimorpha (Lév.) Groposo, Loq.-Leite \& Goés-Neto: S. Vázquez 67: 30.03.1994, Loc. 26.

*Phellinus sarcites (Fr.) Ryvarden: $S$. Vázquez Za29: 03.10.2009, Loc. 30; S. Vázquez Za54: 13.03.2010, Loc. 30; A.P. Crespo Za3: 03.10.2009, Loc. 30. S. Vázquez Zx-11: 28.11.2009, Loc. 22.

\section{Polyporales}

\section{Ganodermataceae}

* Ganoderma aff. lucidum (W. Curt.: Fr.) Karst: N. Martínez Z-50, 28.11.2009, Loc. 22.

Ganoderma curtisii (Berkeley) Murrill: $G$. Guzmán 2179: 23.08.1959, Loc. 9; $G$. Guzmán 6040: 06.08.1967, Loc. 10.

\section{Gloeophyllaceae}

*Gloeophyllum mexicanum (Mont.) Ryv.: S. Vázquez Cg-35: 08.04.2009, Loc. 1.

Gloeophyllum saepiarium (Wulf.: Fr) Fr.: Archundia s.n.: 15.09.1978, Loc. 18.

\section{Polyporaceae}

Antrodia albida (Fr.) Donk: R. Valenzuela 1286: 21.03.1983, Loc. 12.

Climacocystis borealis (Fr.) Kotlava \& Pouzan: P. Ramírez 6-12: 23.07.1981, Loc. 2.

Coriolopsis brunneoleuca (Berkeley) Ryvarden: Vázquez \& Palacios 28: 23.10.1993, Loc. 29.

Coriolopsis gallica (Fr.) Ryvarden: $G$. Guzmán XP-2352: 03.08.1960, Loc. 16.

Cryptoporus volvatus (Pk.) Shear:. $G$. Guzmán s.n.: 23.08.1959, Loc. 7.

Hexagonia hydnoides Sw.: Fr.: G. Guzmán 
Apéndice. Continuación.

s.n.: 02.08.1959, Loc. 12; R. Valenzuela 1285: 21.03.1983, Loc. 12.

Hydnopolyporus fimbriatus (Hook in Kunth) O. Fidalgo: G. Guzmán 2336: 03.07.1960, Loc. 11.

Laetiporus sulphureus (Bull.: Fr.) Murrill: P. Velázquez 414: 22.07.1973, Loc. 4.

Lentinus crinitus (L.) Fr.: G. Guzmán s.n.: 02.08.1959, Loc. 11; J.L. Ursúa s.n.: 28.06.1959, Loc. 13; A. Lerma s.n.: 16.09.1979, Loc. 18; S. Vázquez Za39: 03.10.2009, Loc. 30; S. Vázquez Ap-41: 31.03.2010, Loc. 23.

Lentinus strigosus Fr.: G. Guzmán s.n.: 03.06.1960, Loc. 10.

Lenzites betulina (Fr.) Fr.: G. Rodríguez s.n.: 21.06.1983, Loc. 12; S. Vázquez 84b: 22.10.1994, Loc. 24.

Polyporus leprieurii Mont.: F. Ventura s.n.: 28.08.1970, Loc. 21.

*Postia aff. caesia (Schrad.) P. Karst.: S. Vázquez Zx-32: 28.11.2009, Loc. 22. A.P. Crespo Zc-7: 28.11.2009, Loc. 22; N. Martínez Z-5: 28.11.2009, Loc. 22; N. Martínez Z-27: 28.11.2009, Loc. 22.

*Perenniporia scutellata Murrill: A. P. Crespo Zc-6, 28.11.2009, Loc. 22.

Phaeolus schweinitzii (Fr.) Pat.: H. Ríos s.n.: 06.08.1967, Loc. 12; H. Frías s.n.: 06.08.1967, Loc. 10.

Pleurotus smithii Guzmán: A. Lerma s.n.: 16.09.1979, Loc. 18.

Polyporus arcularius (Batsch) Fr.: $R$. Valenzuela s.n.: 21.03.1983, Loc. 12; G. Guzmán 2183: 23.08.1954, Loc. 10; G. Guzmán XP-1998: 28.06.1959, Loc. 13; F. Ventura 2218: 28.06.1970, Loc. 21; F. Ventura 456, 30.01.1970, Loc. 20.

*Polyporus aff. arcularius (Batsch) Fr.: $S$. Vázquez Za61: 13.03.2009, Loc. 30.

Polyporus tenuiculus (Beauv.) Fr.: L.
López s.n.: 07.1966, Loc. 12; Vázquez \& Palacios 27: 23.10.1993, Loc. 29.

*Polyporus tricoloma Mont.: S. Vázquez Za16: 03.10.2009, Loc. 30; S. Vázquez Ap-31: 31.03.2010, Loc. 23.

Rigidoporus microporus (Fr.) Overheem: Vázquez \& Palacios 11: 23.10.1993, Loc. 29; Vázquez \& Palacios 32: 24.10.1993, Loc. 27; S. Vázquez 98: 23.10.1994, Loc. 32.

Trametes elegans (Spreng.: Fr.) Fr.: R. Torres 188: 23.12.1979, Loc. 5; A. Lerma s.n.: 16.09.1979, Loc. 18; S. Vázquez 98-8: 10.04.1998, Loc. 31 .

Trametes hirsuta (Wulf.: Fr.) Pilat.: $G$. Rodríguez 1478: 21.03.1983, Loc. 12.

Trametes maxima (Montagne) David \& Rajchenberg: F. Brizuela 363: 25.07.1968, Loc. 17.

Trametes pavonia (Hook) Ryvarden: Vázquez \& Palacios 13: 23.10.1993, Loc. 29; Vázquez \& Palacios 33, 23.10.1993, Loc. 28.

Trametes versicolor (L.: Fr.) Pilat: A.M. Pascoe 1: 18.12.1965, Loc. 12; F. Brizuela s.n.: 25.07.1968, Loc. 17; S. Vázquez Zal: 03.10.2009, Loc. 30; N. Martínez Ap-6: 31.03.2010, Loc. 23; A.P. Crespo Zc-21: 13.03.2010, Loc. 22; S. Vázquez Zx-96: 13.03.2010, Loc. 22; S. Vázquez Cg-27: 08.04.2009, Loc. 1; S. Vázquez Cg-66: 07.11.2009, Loc. 1.

Trametes villosa (Fr.) Kreisel: Mille 173: 06.08.1967, Loc. 11; S. Vázquez Za37: 03.10.2009, Loc. 30; S. Vázquez Za49: 13.03.2010, Loc. 30.

*Trichaptum abietinum (Dicks.) Ryvarden: S. Vázquez Zx-90: 13.03.2010, Loc. 22; $S$. Vázquez Cg-43: 07.11.2009, Loc. 1.

*Trichaptum aff. polycystidiatum (Pilat) Y.C. Dai: S. Vázquez Zx-13: 28.11.2009, Loc. 22. 
Apéndice. Continuación.

Trichaptum biformis (Fr. in K1.) Ryvarden: A. López s.n.: 04.09.1966, Loc. 12; G. Guzmán 5398: 04.09.1966, Loc. 12.

*Trichaptum fuscoviolaceum (Ehrenb.) Ryvarden: A.P. Crespo Zc-8: 28.11.2009, Loc. 22.

*Trichaptum imbricatum Y.C. Dai \& B.K. Cui: S. Vázquez Zx-13a: 28.11.2009, Loc. 22.

Trichaptum sector (Ehrenb.: Fr.) Kreisel: F. Ventura 451: 24.01.1970, Loc. 21; F. Ventura 8780: 06.08.1973, Loc. 19.

\section{Russulales}

Auriscalpium vulgare S.F. Gray: Archundia 660: 12.11.1978, Loc. 18; S. Vázquez Cg2: 08.04.2009, Loc. 1.

\section{Stereales}

\section{Meruliaceae}

*Merulius tomentosus Burt: S. Vázquez Ap33: 31.03.2010, Loc. 23.

\section{Stereaceae}

Cymatoderma caperatum (Berk. et Mont.) Reid: G. Salinas 13: 04.09.1966, Loc. 12. *Stereum aff. gausapatum (Fr.) Fr.: $S$. Vázquez Cg-4: 08.04.2009, Loc. 1.

*Stereum aff. hirsutum: S. Vázquez Zx-99: 13.03.2010, Loc. 22.

Stereum complicatum (Fries) Fries: $P$. Domínguez 49: 06.08.1967, Loc. 12; R. Cruz s.n.: 29.07.1963, Loc. 15; G. Guzmán 6034: 06.08.1967, Loc. 11; G. Guzmán 6038: 06.08.1967, Loc. 10; S. Vázquez Ap-2: 06.11.2009, Loc. 23; S. Vázquez Ap-38: 31.03.2010, Loc. 23; $N$. Martínez Ap-1: 31.03.2010, Loc. 23; S. Vázquez Zx-34: 28.11.2009, Loc. 22; A.P. Crespo Zc-4: 28.11.2009, Loc. 22; N. Martínez Z-4: 28.11.2009, Loc. 22; S. Vázquez Zx-49: 13.03.2010, Loc. 22; $S$. Vázquez Zx-85: 13.03.2010, Loc. 22; S. Vázquez Cg-34: 08.04.2009, Loc. 1.
Stereum gausapatum (Fries) Fries: M.S. Fernández 157: 09.1967, Loc. 12.

Stereum hirsutum (Wild: Fr.) S.F. Gray: $R$. Cruz s.n.: 29.07.1963, Loc. 15; S. Vázquez Cg-25: 08.04.2009, Loc. 1.

*Stereum rosea: S. Vázquez Cg-5: 08.04.2009, Loc. 1.

\section{Thelephorales}

\section{Phanerochaetaceae}

*Byssomerulius corium (Pers.) Ginns: $N$. Martínez Z-14: 28.11.2009, Loc. 22; S. Vázquez Zx-53: 13.03.2010, Loc. 22; S. Vázquez Zx-61: 13.03.2010, Loc. 22; A.P. Crespo Zc18: 13.03.2010, Loc. 22.

*Phanerochaetaceae aff. sulphurina: S. Vázquez Cg-29: 08.04.2009, Loc. 1.

*Phanerochaete cremea (Bres.) Parmasto: S. Vázquez Zx-16: 28.11.2009, Loc. 22; A.P. Crespo Zc-3: 28.11.2009, Loc. 22; N. Martínez Z-28: 28.11.2009, Loc. 22.

*Terana coerulea (Lam.) Kuntze: $S$. Vázquez Ap-5: 06.11.2009, Loc. 23; S. Vázquez Zx70: 13.03.2010, Loc. 22.

\section{Tricholomatales}

\section{Cyphellaceae}

Campanophyllum proboscideum (Fr.) Cifuentes \& R.H. Peterson: S. Vázquez Za50: 13.03.2009, Loc. 30.

\section{Mycenaceae}

Mycena leaina (Berk) Sacc.: G. Guzmán s.n.: sin fecha, Loc. 8.

Tricholomataceae

*Clitocybe geotropa (Bull.) Quél.: S. Vázquez Za-53: 13.03.2009, Loc. 30.

Clitocybe gibba (Pers.) P. Kumm: P. Ramírez 4-7: 25.06.1981, Loc. 3.

\section{Phragmobasidiomycetes}

Tremellales

\section{Tremellaceae}

*Tremella aff. mesenterica: $S$. Vázquez Cg48: 07.11.2009, Loc. 1. 
Apéndice. Continuación.

Tremella lutescens Fr.: R. Castañeda s.n.: sin fecha, Loc. 6.

*Tremella mesenterica Schaeff.: N. Martínez
Z-24: 28.11.2009, Loc. 22; S. Vázquez Zx3: 28.11. 2009, Loc. 22; S. Vázquez Cg-1: 08.04.2009, Loc. 1. 